\title{
VARIETIES AND D.G. NEAR-RINGS
}

\author{
by J. D. P. MELDRUM
}

(Received 5th June 1970)

In this note we show that the variety of near-rings generated by d.g. nearrings is the class of all near-rings $R$ satisfying

$$
0 x=x 0=0 \text { for all } x \in R .
$$

This extends a result of $J$. J. Malone (3) on the embedding of near-rings.

A near-ring $R$ is a set with two binary operations, addition and multiplication such that $R$ is a group with respect to addition, a semi-group with respect to multiplication and $x(y+z)=x y+x z$ for all $x, y, z \in R$. If $x \in R$ satisfies $(y+z) x=y x+z x$ for all $y, z \in R$, we say that $x$ is distributive. A distributively generated (d.g.) near-ring is one which is generated as an additive group by its distributive elements.

A variety of near-rings is the class of all near-rings satisfying a given set of laws. Let $X$ be a class of near-rings. Then let $v X$ be the smallest variety containing $\boldsymbol{X}, \mathrm{s} \boldsymbol{X}$ the class of all sub near-rings of $\boldsymbol{X}$ near-rings, $\mathrm{Q} X$ the class of all homomorphic images of $\boldsymbol{X}$ near-rings and $\mathrm{R} \boldsymbol{X}$ the class of all residually $\boldsymbol{X}$ near-rings. For further explanation of these ideas see P. M. Cohn (1).

Let $G$ be a group written additively ( $G$ is not necessarily abelian). Then we can define $T(G)$, the near-ring of all mappings from $G$ to $G$ where addition in $T(G)$ is given by $g(x+y)=g x+g y, g \in G, x, y \in T(G)$, and multiplication in $T(G)$ is the usual product of maps. $T(G)$ contains the following sub near-rings, each containing the next:

$T_{0}(G)$, the set of all mappings preserving the identity of $G$;

$E(G)$ the d.g. near-ring generated by all the endomorphisms of $G$;

$I(G)$ the d.g. near-ring generated by all the inner automorphisms of $G$.

Denote by $O$ the variety of near-rings satisfying (1) and let

$$
\begin{aligned}
& T=\left\{R ; R \cong T_{0}(G) \text { for some group } G\right\}, \\
& I=\{R ; R \cong I(G) \text { for some group } G\}, \\
& F=\left\{R ;|R|<\aleph_{0}\right\} .
\end{aligned}
$$

We will show that $O=v I$, by showing that $O \leqq \mathrm{SQR} I$, from which we can deduce that $O=\operatorname{SQR} I=v I$, using results from P. M. Cohn (1). In (3) J. J. Malone proved that $\boldsymbol{O} \cap \boldsymbol{F}=\mathrm{s}(\boldsymbol{I} \cap \boldsymbol{F})$.

Lemma 1. $O=s T$. 
Proof. $\mathrm{s} T \leqq O$ is immediate. $O \leqq \mathrm{~s} T$ follows immediately from Theorem 1 of J. J. Malone and H. E. Heatherly (4), which states that if $R \in O$, then $R$ can be embedded in $T_{0}(G)$ for any group $G$ such that $G$ contains properly the additive group of $R$.

By using this result, we can restrict our attention to $T_{0}(G)$ where $G$ belongs to a class of groups such that any group can be embedded in a group of this class. By Theorem 11.5.4 of W. R. Scott (5), such a class is that of the simple (non-abelian) groups. So let $G$ be a simple non-abelian group. Let $\left\{\delta_{\lambda} ; \lambda \in \Lambda\right\}$ be the set of all finite subsets of $G, R=T_{0}(G), S=I(G)$. Then A. Fröhlich (2) has established

Lemma 2. Let $r \in R$ and let $\delta_{\lambda}$ be a finite subset of $G$. Then there is an element $s_{\lambda} \in S$ such that $g r=g s_{\lambda}$ for all $g \in \delta_{\lambda}$.

This is essentially result 5.2 of (2). If $G$ is finite, this shows that $R=S$.

For every $\lambda \in \Lambda$, let $S_{\lambda} \cong S$. Denote by $T$ the direct product $\prod_{\lambda \in \Lambda} S_{\lambda}$. Then we will show that $R$ is isomorphic to the homomorphic image of a sub near-ring $U$ of $T$, whose projection into each factor is onto. $U$ is then a subdirect product of the $S_{\lambda}$ and hence lies in RI since $S_{\lambda} \in I$ (see P. Cohn (1) for a proof that $R \in \mathrm{R} X$ if and only if $R$ is a subdirect product of $X$ near-rings).

We define a partial ordering on $\Lambda$ by $\lambda \leqq \mu$ if and only if $\delta_{\lambda} \leqq \delta_{\mu}$. Then given $\lambda_{1}, \lambda_{2}$, there is a $\mu$ satisfying $\mu \geqq \lambda_{i}, i=1,2$. Just take $\delta_{\mu}=\delta_{\lambda_{1}} \cup \delta_{\lambda_{2}}$. Let $t \in T$. Call $t$ eventually constant if given $g \in G, \lambda \in \Lambda$, there is a $\mu \geqq \lambda$ such that for all $\eta \geqq \mu, g t(\eta)=h$ is independent of $\eta$. We are here considering $t(\eta)$ as lying in $S$ for all $\eta$. Let $U=\{t ; t \in T$ and $t$ is eventually constant $\}$.

Lemma 3. $U$ is a subdirect product of the $S_{\lambda}, \lambda \in \Lambda$. That is, $U \in \mathbf{R} I$.

Proof. We only need to show that $U$ is a sub near-ring, since the constant elements $t \in T$ satisfying $t(\lambda)=t(\mu)$ for all $\lambda, \mu$ in $\Lambda$ lie in $U$ and so the projection of $U$ into each factor will be onto. Let $u, v \in U$, and let $g \in G, \lambda \in \Lambda$ be given. Then we have $\mu_{1} \geqq \lambda, \mu_{2} \geqq \lambda$ such that if $\eta \geqq \mu_{1}, g u(\eta)=h, h$ independent of $\eta$, if $\eta \geqq \mu_{2}, g v(\eta)=k, k$ independent of $\eta$. Let $\mu \geqq \mu_{i}, i=1,2$. Then for $\eta \geqq \mu, g u(\eta)=h, g v(\eta)=k$ and so $g(u+v)(\eta)=h+k$. Hence $u+v \in U$. Also we have $\mu_{3} \geqq \lambda$ such that if $\eta \geqq \mu_{3}, h v(\eta)=l, l$ independent of $\eta$. Let $\mu^{\prime} \geqq \mu_{i}$, $i=1,3$. Then for $\eta \geqq \mu^{\prime}, g(u v)(\eta)=(g u(\eta)) v(\eta)=h v(\eta)=l, l$ independent of $\eta$. Hence $u v \in U$. Finally it is obvious that $u \in U$ gives $-u \in U$ and that the additive and multiplicative identities are in $U$.

We are interested in what happens "eventually". So we define an equivalence relationship on the elements of $U$ as follows. Let $u, v \in U$. Then $u \sim v$ if given $g \in G, \lambda \in \Lambda$, there is a $\mu \geqq \lambda$ such that for all $\eta \geqq \mu, g u(\eta)=g v(\eta)$. The fact that $\sim$ is symmetric and reflexive is immediate. If $u \sim v, v \sim w$, then given $g \in G, \lambda \in \Lambda$, there is a $\mu_{1} \geqq \lambda$ such that for all $\eta \geqq \mu_{1}, g u(\eta)=g v(\eta)$, and a $\mu_{2} \geqq \lambda$ such that for all $\eta \geqq \mu_{2}, g v(\eta)=g w(\eta)$. So if $\mu \geqq \mu_{i}, i=1,2$, then for all $\eta \geqq \mu, g u(\eta)=g v(\eta)=g w(\eta)$. Hence $u \sim w$. We have 
Lemma 4. $\sim$ is an equivalence relationship on $U$.

We next show that $\sim$ is compatible with multiplication and addition in $U$.

Lemma 5. Let 0 be the additive identity in $U$, and let

$$
N=\{u ; u \in U, u \sim 0\} .
$$

Then $N$ is an ideal in $U$ and $\sim$ is compatible with multiplication and addition in $U$.

Proof. Let $v, w \in U, u \in N$. Let $g \in G, \lambda \in \Lambda$. Then as in the proof of Lemma 3, there is a $\mu \geqq \lambda$ such that $g w(\eta), g v(\eta)$ and $g u(\eta)$ are constant and $g u(\eta)=0$ for all $\eta \geqq \mu$. So $g(-v+u+v)(\eta)=-g v(\eta)+g u(\eta)+g v(\eta)=0$, i.e. $-v+u+v \in N$. We now need to show that $u w \sim 0$ and $(w+u) v-w v \sim 0$. If $\eta \geqq \mu$, then $g u w(\eta)=g u(\eta) w(\eta)=0 w(\eta)=0$. So $u w \sim 0$. If $g w(\eta)=k$ for $\eta \geqq \mu$, there is a $\mu_{1} \geqq \lambda$ such that $k v(\eta)$ is constant for $\eta \geqq \mu_{1}$. Then if $\mu_{2} \geqq \mu_{1}$, $\mu_{2} \geqq \mu$, we have

$$
g(w+u) v(\eta)=(g w(\eta)+g u(\eta)) v(\eta)=k v(\eta)=g w v(\eta) \text { as } g u(\eta)=0,
$$

for $\eta \geqq \mu_{2}$. So $(w+u) v-w v \sim 0$ and $N$ is an ideal in $U$. Finally if $u \sim v$, then by the definition of $\sim$ it is immediate that $u-v \sim 0$. Hence $\sim$ is compatible with multiplication and addition in $U$.

We can now prove our result.

Theorem 6. $O=S Q R I$.

Proof. Let $\theta: R \rightarrow U / N$, which is a near-ring by Lemma 5 , be given by $r \theta=u+N$ where $u(\lambda)$ is given by $g r=g u(\lambda)$ for all $g \in \delta_{\lambda}$. This is possible by Lemma 2. Given $g \in G, \lambda \in \Lambda$, let $\mu$ be such that $g=\delta_{\mu}$ and $\mu \geqq \lambda$, e.g. $\delta_{\mu}=\delta_{\lambda} \cup\{g\}$. Then if $\eta \geqq \mu, g u(\eta)=g r$ is constant. So $u \in U$. But $u(\eta)$ is not uniquely defined. Suppose that $u^{\prime}(\eta)$ also satisfies $g r=g u^{\prime}(\eta)$ if $g \in \delta_{\eta}$. Then $g\left(u-u^{\prime}\right)(\eta)=0$ if $g \in \delta_{\eta}$ and hence for all $\eta \geqq \mu$ as defined above So $u+N=u^{\prime}+N$ and $r \theta$ is uniquely defined.

Let $r, s \in R, r \theta=u+N, s \theta=v+N$. Given $g \in G, \lambda \in \Lambda$, let $\delta_{\mu}=\delta_{\lambda} \cup\{g\}$. Then $g u(\eta)=g r$ and $g v(\eta)=g s$ for all $\eta \geqq \mu$. So

$$
g(u+v)(\eta)=g u(\eta)+g v(\eta)=g r+g s=g(r+s)
$$

Hence if $(r+s) \theta=w$, then $w+N=u+v+N$.

Now let $g \in G, g r=h, \lambda \in \Lambda$. Define $\mu$ by $\delta_{\mu}=\delta_{\lambda} \cup\{g, h\}$. Then

$$
g u(\eta)=g r, g v(\eta)=g s \text { and } g(u v)(\eta)=(g u(\eta)) v(\eta)=h v(\eta)=h s=g r s
$$

for all $\eta \geqq \mu$. So if $(r s) \theta=w$, then $w+N=u v+N$. This shows that $\theta$ is a homomorphism.

Suppose that $r \theta=N+u$ and $u \in N$. Then given $g \in G, \lambda \in \Lambda$, let $\mu_{1}$ be defined by $\delta_{\mu_{1}}=\delta_{\lambda} \cup\{g\}$. We have $g u(\eta)=g r$ for all $\eta \geqq \mu_{1}$. But $u \sim 0$. So given $g \in G, \mu_{2} \in \Lambda$, there is a $\mu_{2} \geqq \mu_{1}$ such that $g u(\eta)=0$ for all $\eta \geqq \mu_{2}$.

E.M.S. $-S$ 
But $\eta \geqq \mu_{2}$ gives us $g r=g u(\eta)=0$. This is true for all $g \in G$. So $r=0$, and $\theta$ is an isomorphism.

Finally if $u \in U$, given $g \in G, \lambda \in \Lambda$, there is a $\mu \geqq \lambda$ such that $g u(\eta)=h$ is constant for $\eta \geqq \mu$. In particular if $\mu_{1}$ is defined by $\delta_{\mu_{1}}=\delta_{\mu} \cup\{g\}$, we have $g u(\eta)=h$ for $\eta \geqq \mu_{1}$. Define $r \in R$ by $g r=h$, and let $v+N=r \theta$. Then for $\eta \geqq \mu_{1}, g v(\eta)=g r=h=g u(\eta)$. So $v+N=u+N$. Hence $\theta$ is onto and $R \cong U / N$.

By Lemma 3, $U \in \mathrm{R} I$ and so $U / N \in \mathrm{QR} I$. Hence $R \in \mathrm{QR} I$ and by the remarks made after Lemma $1, T \leqq Q R I$. By the remarks made just before Lemma 1 and Lemma 1 itself, $O=$ SQR $I$, thus finishing the proof of the theorem.

This extends J. J. Malone's result (3) that $O \cap F=\mathrm{s}(I \cap F)$ in the sense that we remove the $F$, but have to replace $I$ by QR $I$. It would be interesting to know if we can get rid of $Q R$ or replace it by something simpler.

I am grateful to Professor J. J. Malone for letting me have a copy of his paper (3) before publication.

\section{REFERENCES}

(1) P. M. CoHN, Universal Algebra (Harper and Row, 1965).

(2) A. FröHLICH, The near-ring generated by the inner automorphisms of a finite simple group, J. London Math. Soc. 33 (1958), 95-107.

(3) J. J. Malone, A near-ring analogue of a ring embedding theorem, J. Algebra 16 (1970), 237-238.

(4) J. J. Malone and H. E. Heatherly, On some near-ring embeddings, Quart. J. Math. Oxford Ser. (2), 20 (1969), 81-85.

(5) W. R. Scort, Group Theory (Prentice Hall, 1964).

Mathematical Institute

Chambers STREeT

EDINBURGH 1 